\title{
Synthesis and Characterization of Biogenic Iron Sulfide Nanoparticles in Cancer and Other Biomedical Applications: A Review
}

\author{
Hemant Kumar ${ }^{2,3,4}$, Tarun Mohan ${ }^{3}$, Pramod Kumar' ${ }^{1}$ and Balaram \\ Pani* \\ ${ }^{1}$ Department of Chemistry and Chemical Science, School of Physical and Material \\ Sciences, Central University of Himachal Pradesh, Dharamshala, India \\ ${ }^{2}$ Department of Chemistry, Ramjas College, University of Delhi, India \\ ${ }^{3}$ Department of Chemistry, University of Delhi, India \\ ${ }^{4}$ Bhaskaracharya College of Applied Sciences, Department of Chemistry, University \\ of Delhi, India
}

*Corresponding Author: Balaram Pani, Bhaskaracharya College of Applied

Sciences, Department of Chemistry, University of Delhi, India.
Received: October 01, 2021

Published: February 04, 2022

(C) All rights are reserved by Balaram Pani., et al.

\begin{abstract}
This review focuses on recent breakthroughs in the physicochemical approaches and biological approaches of metallic iron, iron sulfides, and iron oxide nanoparticles for a variety of applications. The range of kinds, topologies, and physicochemical features of nano-sized iron sulfides has piqued researchers' curiosity. Furthermore, this study examines the medicinal, environmental, and technical uses of biogenically synthesized NPs, as well as the hurdles that must be overcome in order to optimize the environmentally friendly production of these critical nanoparticles. FeS NPs have a good effect on biological activity due to their ease of production, magnetic properties, biocompatibility, and biodegradability. Also canvased are the FeS NPs nanoparticle-specific biomedical applications in cancer treatments. The goal of this review is to discuss the synthesis, characteristics, and uses of nano sized FeS NPs in biomedical domains, revealing that they have significant promise for enhancing human health and excellence of life.
\end{abstract}

Keywords: Iron Sulfides; Surface Modification; Biocompatibility; Cancer Therapy; Photothermal Therapy; Biosensors; Antifungal Agents; Enzyme-Like Catalysis

\section{Introduction}

Nanoscience can be explained as the study of the property of matter and structure on a nanoscale between $1-100 \mathrm{~nm}$. The several properties of the matter like interfacial and surface chemistry useful in nanotechnology's applications. The high surface-to-volume ratio useful in catalysis, it enhances the chemical properties of nanomaterials. Mechanical properties boost the strength hardness in lightweight nanomaterial and Nanocomposites [1]. It purely depends on the unique and size-dependent property of solid matter.

The biomedical nanotechnology is an interdisciplinary field of study that spans health, science and engineering. It also has a wide variety of molecular diagnostic, molecular imaging, and targeted treatment applications. The amalgamation of nanotechnology with molecular biology has begotten a new research area or sector that transforms biomedical research by providing specific imaging techniques, nano-robotics, and nano-devices, etc [2-3]. Nanomaterials with a spherical shape are very useful in the field of nanomedicine as they are similar in dimension to biological macromolecules, and smaller than cellular organelles. The nano-sized particles such as iron oxide and quantum dots have magnetically, optically, or structural properties that differ from the bulk materials. These biocompatible nanoparticles have a high affinity and selectivity for sick cells and organ-like malignant tumors. Nanopar- 
ticles can also be linked with bio-targeting ligands like peptides and monoclonal antibodies. They have a variety of advantages for medical applications, including high surface areas and functional groups, cross-linking to various diagnostic and therapeutic agents such as magnetic, radio-isotropic, and optical, and encapsulation of bioactive agents such as fluorophores and drugs, among others. And quantum properties, which are used for conjugating multiple diagnostics like magnetic, radio isotropic and optical, and therapeutic agents. Because of the enhanced permeability effect (EPR), the nanomaterial $(<100 \mathrm{~nm})$ has the capacity to acquire and gather tumor locations, which aids in early tumor identification and treatment. After the interaction, they should maintain their physical properties after modification and remain non-toxic. Nanomaterials have unique properties that show their ultra-small dimension. And it is designed to assist the transport of diagnostic or therapeutic agents through biological barriers to acquire access within cells. And arbitrate molecular interactions and trace molecular changes in an intuitive and out-turn manner. The main applications of nanomaterials in biology are drug delivery [4-5], bio-detection of pathogens [6], fluorescent and biological labelling, probing of DNA structure [7], tissue engineering [8,9], tumor ruination by hyperthermia [10], MRI contrast enhancement [11], detection of protein [12], etc.

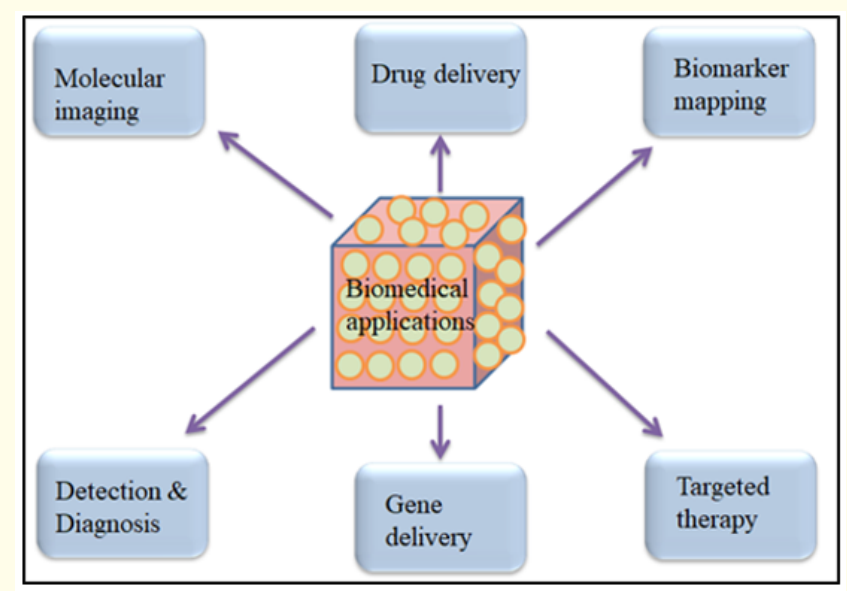

Figure 1: Biomedical application of nanotherapeutics.

Metallic nanoparticles have attracted a lot of interest in the past few years because of their unusual physical, chemical, and biological features, which are linked to their large surface-to-volume ratio and quantum confinement effects. Chalcogenides of transition metals have sparked a lot of attention recently, and their characteristics [13] have led to their usage in superconductors [14], magnetic semiconductors [15], photovoltaics [16], sensors [17], thermoelectrics, optoelectronic behavior, their indirect bandgaps, and the catalysts might absorb visible light, which is plentiful in solar radiation. Iron chalcogenides have been identified as an important and promising nanomaterial among the transition metal chalcogenides [17-23]. The structural features, semiconducting characteristics, magnetic characteristics, and biocompatibility are all reasons for this interest [18-24]. They have also been employed in biological applications including antibacterial activity [24], magnetic resonance imaging (MRI), photothermal therapy, magnetically guided drug delivery, and biocatalysis [21] based on these features. Other benefits of iron chalcogenides are their ease of production and ultra-small size [25]. There are several types of iron sulfide, including mackinawite $(\mathrm{FeS})$, troilite $(\mathrm{FeS})$, greigite $\left(\mathrm{Fe}_{3} \mathrm{~S}_{4}\right)$, pyrrhotite $\left(\mathrm{Fe}_{1-\mathrm{x}}\right.$ $\mathrm{S}$, frequently $\mathrm{Fe}_{7} \mathrm{~S}_{8}$ and $\mathrm{Fe}_{8} \mathrm{~S}_{9}$ ), and pyrite is a mineral that is found in cubic form $\left(\mathrm{FeS}_{2}\right)$. While some exclusively have Fe (II), others, like greigite, have both Fe (II) and Fe (III). Based on its characteristics, the iron sulfide ( $\mathrm{FeS}$ ) nanostructure has a greater potential for biological applications $[23,25]$. Because of their biological action, xanthene derivatives are extremely essential molecules in medicine. They are also used as dye components in lasers, pigments, and fluorescent biomolecule detection systems. Because of the pyran ring, 9-aryl-1, 8-dioxooctahydroxanthenes, and 14-substituted$14 \mathrm{H}$ dibenzoxanthene derivatives are found in a variety of natural compounds and are employed as multimodal synthons [25].

FeS nanoparticles (NPs) are being used in biological and catalysts for organic synthesis and many more applications such as the interactions of FeS NPs with proteins and enzyme is critical. In biological processes, proteins and enzymes play a crucial role [26]. FeS is an innocuous, widely distributed mineral that acts as a precursor to the more stable iron FeS minerals greigite and pyrite. As a consequence, chlorinated organic compounds (COCs), heavy metals, arsenic, selenium, and other inorganic and organic contaminants in groundwater and soil have been remedied using FeS. Because they combine with $\mathrm{Hg}$ to produce an insoluble product, $\mathrm{HgS}$, many naturally occurring sulfide minerals have been identified as the primary sink for $\mathrm{Hg}$ in the atmosphere. Previous research suggested that adsorption, inclusion, precipitation, and surface complexation might be involved in the interaction between $\mathrm{Hg}$ (II) and FeS. Furthermore, FeS nanoparticles were produced and proven 
to be efficient in collecting $\mathrm{Hg}$, demonstrating a much higher sorption capacity than that of the other sorbents FeS NPs are smaller, have a larger specific surface area, have more active sorption sites, and stronger surface reactivity. FeS particles are nanoscale materials are projected to provide increased soil deliverability and adsorption capacity. They have a tendency to clump together and develop fast to micrometer or millimeter scale particles, reducing their mobility and chemical responsiveness. Previous investigation has shown that carboxymethyl cellulose (CMC), an improved natural polysaccharide, may efficiently stabilize FeS NPs via steric hindrance and electrostatic repulsion, resulting in very stable CMCFeS nanoparticles [27].

\section{Iron sulfide nanoparticle synthesis}

There are two types of synthetic techniques for FeS nanostructured materials and conventional production techniques for bare nano-sized FeS particles; physicochemical approaches and biological approaches. Chemical precipitation is the standard approach for making bare nanoscale FeS. It proposes that FeS nanoparticles might be produced by co-precipitating $\mathrm{Fe}^{2+}$ and $\mathrm{S}^{2-}$ ions in an aqueous phase under anaerobic circumstances while stirring constantly [28]. Iron $\left(\mathrm{Fe}^{2+}\right)$ source precursors include iron sulfate heptahydrate, ferrous chloride $\left(\mathrm{FeCl}_{2}\right)$, and ammonium ferrous sulfate while thiourea $\left(\mathrm{CH}_{4} \mathrm{~N}_{2} \mathrm{~S}\right)$, Sodium dithionite $\left(\mathrm{Na}_{2} \mathrm{~S}_{2} \mathrm{O}_{4}\right)$ and sodium sulfide $\left(\mathrm{Na}_{2} \mathrm{~S} \cdot 9 \mathrm{H}_{2} \mathrm{O}\right)$ were used to provide $\mathrm{S}^{2-}$ ions. The co-precipitation of $\mathrm{FeS}$ in the aqueous phase under anoxic conditions is the most frequent traditional physicochemical technique.

$\mathrm{Fe}^{2+}+\mathrm{S}^{2-} \rightarrow \mathrm{FeS}$

FeS NPs synthesize by reacting the ammonium ferrous sulfate (Mohr's salt) with sodium sulfide at room temperature under $\mathrm{N}_{2}$ atmosphere, and a black precipitate formed.

FeS NPs can be synthesized by the biosynthesis method (biological process), recent studies have lots of interest due to its "green synthesis" and environmentally friendly nature. Sulfatereducing bacteria (SRB) may create sulfide by using sulfur species (Sulphate, thiosulfate, etc.) as a terminal electron acceptor. Ferric reducing bacteria (FRB) reduce $\mathrm{Fe}^{3+}$ ions; and $\mathrm{S}^{2-}$ ions and $\mathrm{Fe}^{2+}$ ions respectively to produce FeS NPs [29-31]. FeS NPs are produced by the Acidiphilium cryputum JF-5 and SRB (Desulfovibrio vlugaris miyazaki), and it is black in color and its XRD pattern resembles the mackinawite $\left(\mathrm{FeS}_{0.9}\right)$ [32]. Moreover, FeS nanoparticles synthesized by Shewanella species; can instantaneously reduce $\mathrm{Fe}^{3+}$ ions, and sulfur works as a terminal electron acceptor when lactate worked as an electron acceptor [33].

Microwave-assisted procedures have several gains over traditional heating methods, including decreased time of reaction, narrower nanoparticle size dispersion, and greater pureness. Ethylene glycol $\left(\mathrm{CH}_{2} \mathrm{OH}\right)_{2}$ is an excellent solvent for microwave-assisted technique because of its large dipole moment. iron sulfate heptahydrate, PVP-K30, and sulfur (S) in ethylene glycol can be microwaved in a nitrogen $\left(\mathrm{N}_{2}\right)$ gas environment to make FeS/ $\mathrm{FeS}_{2}$ microspherolites [34]. Although this new methodology appears to be more desired, the clump phenomena do not seem to have improved.

Bala and co-workers designated the conventional sonochemical approaches to synthesize the FeS nanoparticles. To begin, $\mathrm{Na}_{2} \mathrm{~S} \cdot 9 \mathrm{H}_{2} \mathrm{O}$ was dissolved in distilled water (DW). And then, ferrous sulfate $\left(\mathrm{FeSO}_{4} .7 \mathrm{H}_{2} \mathrm{O}\right)$ was dissolved separately in DW; and a 1:1 mole ratio of polyethylene glycol while the sodium sulfide solution was being continuously sonicated for 30 minutes, Triton-X surfactant was added dropwise to the solution. After adding PVP, the solution was stirred for another 30 min using an ultrasound [35,36].

\section{Synthesis of the modified Fes nanoparticles}

To generate FeS nanoparticles with normalized particle shape and size distribution, several particle stabilizing approaches have been investigated. SRB-assisted manufacturing, Reverse micelle, polymer-stabilized wet-chemical synthesis, high-energy mechanical milling, and poly(amidoamine) dendrimer stabilization are among these methods. The latest research has demonstrated that adding polymeric stabilizers to the FeS particle manufacturing process, such as starch and $\mathrm{CMC}$, can help with size control in aqueous solutions. Using CMC as a stabilizer, a unique class of stabilized FeS nanoparticles has been developed. This may efficiently regulate the nucleation and progress of nanoparticles by combining electrostatic repulsion and steric hindrance. A stabilizer called CMC or chitosan was utilized to prevent the FeS NPs from aggregating, and these fabricated nanoparticles performed well in dispersion. FeS NPs have a tendency to clump together and develop fast to micrometer or millimeter scale particles, reducing their mobility and chemical responsiveness. In addition to CMC, several macromolecular biomaterials with similar physicochemical characteristics have sparked attention, including cyclodextrin (CD), starch, and polysaccharide sodium alginate (SA) $[34,37,38]$. 
Applications of Fes nanoparticles

\section{Photothermal therapy}

Photothermal therapy (PTT) is a non-invasive therapeutic method are recently has received a lot of interest $[39,40]$. Photoabsorbing nanoparticles generate heat through uninterrupted laser irradiation, killing cancer cells while leaving healthy tissue untouched [41]. Imaging guided PTT, for example, might not only show sizes, tumor locations, and forms to guarantee that tumors are effectively exposed to the laser during therapy, but it could also indicate the ideal moment for laser irradiation when the photothermal agent reaches a top-level in the tumor. As a consequence, the photothermal characteristics of iron sulfide (FeS) must be taken into consideration. The near-infrared absorption of FeS nanoplates that have been PEGylated (FeS-PEG) is quite high. The multi-enzyme activities, severe environmental resistance, storage strength, and vital advantages of nanomaterials are shown by Infrared thermal imaging, suggesting that excellent replacements for natural enzymes are possible. Iron sulfide's enzymatic activity has gotten a lot of attention. The peroxidase-like activity was discovered in FeS nanosheets in 2010. In the existence of $\mathrm{H}_{2} \mathrm{O}_{2}$, FeS suspensions were demonstrated to catalyze the oxidation of peroxidase substrates $\operatorname{TMB}\left(3,3,3,5,5^{\prime}\right.$-Tetramethylbenzidine $)$ to yield a blue color substance [42]. FeS-PEG outperforms other known iron oxides in terms of photothermal transformation efficiency [43].

\section{Enzyme-like catalysis}

FeS clusters are required as cofactors by several proteins and enzymes that execute redox reactions and control oxidative stress. As a result, FeS nanoparticles are projected to serve as enzymes and conduct comparable catalysis. Iron sulfide has been demonstrated to successfully activate peroxymonosulfate ( $\mathrm{PMS} \mathrm{HSO}_{5}{ }^{-}$) or persulfate ( $\mathrm{PS}, \mathrm{S}_{2} \mathrm{O}_{8}{ }^{2-}$ ) to create sulfate radicals in previous research [44]. Since the discovery of intrinsic peroxidase activity in iron oxide nanoparticles in 2007 [45], it has been assumed that iron sulfide possesses comparable capabilities. Iron sulfide catalyzes a variety of reactions such as High catalytic activity, extreme environmental resistance; multi-enzyme activities, storage stability, and the inherent benefits of nanomaterials all contribute to the advancement of biomedicine, implying that good substitutes for natural enzymes exist. Iron sulfide's enzymatic activity has been extensively studied. FeS nanosheets were discovered to have peroxidase-like activity in 2010.
In 2020, it was observed that light irradiation of FeS NPs might increase intrabacterial ROS levels in bacteria. This was discovered to be FeS NPs' principal antibacterial mechanism [24].

\section{Cancer therapy}

Iron is a vital nutrient that affects a variety of bodily activities, including energy metabolism, hematopoiesis, oxygen metabolism, muscular function, and more. On the other side, iron is important in the development of cancer and cancer-related disorders. Iron is a potent driver of cancer development, invasion, and metastasis in previous research [46]. Furthermore, iron homeostasis is frequently disrupted in cancer patients, making iron deficiency anemia a common consequence. However, in terms of iron speciation, cancer-related iron distortion is not entirely understood $[47,48]$. The ferrous-sulfide-like iron and ferritin chemical forms of iron identified in the tumors suggest that iron sulfide might be utilized to treat cancer [49]. FeS NPs are synthesized by the co-precipitation method and are used in hyperthermia, opening up new possibilities for multimodal anticancer treatments [50]. High dosages of FeS are harmless and efficacious in mice using PEGylated FeS (FeS-PEG) NPs as nanoagents for in-vivo MRI-targeted photothermal cancer therapy. FeS might be used in the clinical for MRI as well as PTT, according to the study [51]. The high effectiveness of iron sulfide nanosheets for MRI- targeted photothermal and chemodynamic synergistic therapy has paved the way for future clinical trials of inorganic iron sulfide. Due to the localized heat generated by PTT (synergistic photothermal therapy) from the defect-rich structure, which could cause overproduction of $\mathrm{H}_{2} \mathrm{O}_{2}$ by the Fenton process, and the formed $\cdot \mathrm{OH}$ could hinder tumor growth and reappearance after PPT, $\mathrm{Fe}_{3} \mathrm{~S}_{4}$ TNSs have high proficiency for MRI guidance and can achieve CDT (chemodynamic therapy) and PTT. As a consequence, we were able to create a high-efficiency inorganic theranostic platform that was effectively evacuated from the body. This will open the path for the future development of inorganic substances like iron sulfide for medicinal purposes [52].FeS-Dox@ bLf NZs were shown to be not only effective in treating and managing breast cancer with minimum adverse effects in vitro and in vivo, but also to give a clear method to synchronize diverse treatments, including chemotherapy, PDT, and so on [53].

\section{Biosensors}

The FeS nanostructure holds the scope for increased biosensing and biocatalysis applications. The sheet-like FeS nanostructures' 
capacity to activate the oxidation of organic compounds to create a color shift is backed up by their appealing functionality of an inbuilt peroxidase-like activity. This $\mathrm{H}_{2} \mathrm{O}_{2}$ sensor was also more sensitive than others that used spherical FeS nanoparticles. Compared to the HRP, the mimic peroxidase was more stable when unprotected to high temperatures and $\mathrm{pH}$ extremes. The sheet-like FeS has an extensive variety of potential accomplishments in biotechnology, biosensors, and environmental chemistry due to its high stability, simplicity of manufacture, and distinctive characteristics. According to the researchers, the unique sheet-like FeS nanosheets worked as an "artificial peroxidase" in the growth of amperometric transducers and biocatalysts. Because of their natural peroxidaselike activity, FeS has been employed as glucose sensors. Colorimetric techniques, in which cascade reactions constitute the primary mechanism of glucose detection [54], can be used to create glucose sensors.

\section{Antifungal agents}

In in-vitro conditions, iron sulfide NPs show antifungal properties against F. verticillioides; at a considerably lower dosage than the conventional fungicide, FeS-NPs considerably suppressed the development of the test fungus. When the concentration was increased from 10 to $30 \mu \mathrm{g} / \mathrm{ml}$, radial growth inhibition increased gradually. FeS-NPs have the potential to be employed as a future substitute to high-dose organic fungicides as ecologically acceptable seed priming antifungal agents, exclusively in iron-deficient soils [55].

\section{Conclusion}

This study focuses on current advancements in environmentally friendly inorganic NPs used for biomedical applications. Although significant progress has been made in the regulated biogenic production of metallic NPs, there are still certain obstacles to overcome. In the foreseeable future, precise control of particle size, morphological form, and monodispersity is still seen as a critical challenge to address. Due to its high photothermal and magnetic performance, nano-sized iron sulfide has also gained interest due to its large potential in-vivo use, notably in cancer treatment. In summary, we have presented the most current techniques of nanoiron sulfide production, including nano-iron sulfide alterations and characterizations. Remarkably, nano-sized iron sulfides display varied physiochemical characteristics, enzyme-like catalysis, excel- lent stability, and cytocompatibility, which assist their biological applications. A spectrum of nano-iron sulfides has been tested in catalysis, tumor treatment, antibiotic ointments and antifungals, biosensors, and in plants. Furthermore, studying multimodal FeS NPs characteristics in a live organism might lead to improved disease detection and therapy in the future.

\section{Acknowledgements}

We would like to acknowledge Ramjas College, University of Delhi, 110007 and Bhaskaracharya College of Applied Sciences, University of Delhi, Delhi-110075.

\section{Conflict of Interest}

The authors state that they have no financial or other conflicts of interest.

\section{Bibliography}

1. Mobasser S., et al. "Review of Nanotechnology Applications in Science and Engineering". Journal of Civil Engineering and Urbanism 6.4 (2016): 84-93.

2. Wang S., et al. "Antigen/Antibody Immunocomplex from CdTe Nanoparticle Bioconjugates". Nano Letters 2.8 (2002): 817822 .

3. Berry CC., et al. "Functionalisation of magnetic nanoparticles for applications in biomedicine". Journal of Physics D: Applied Physics 36.13 (2003): 198.

4. Mah C., et al. "Microsphere-mediated delivery of recombinant AAV vectors in vitro and in vivo". Molecular Therapy 1.5 (2000): S239-S242.

5. Pantarotto Davide, et al. "Immunization with peptide-functionalized carbon nanotubes enhances virus-specific neutralizing antibody responses". Chemistry and Biology 10.10 (2003): 961-966.

6. Edelstein, R. L., et al. "The BARC biosensor applied to the detection of biological warfare agents". Biosensors and Bioelectronics 14.10-11 (2000): 805-813.

7. Nam JM., et al. "Nanoparticle-based bio-bar codes for the ultrasensitive detection of proteins". Science 301.5641 (2003): 1884-1886. 
8. Mahtab R., et al. "Protein-sized quantum dot luminescence can distinguish between" straight"," bent", and" kinked" oligonucleotides". Journal of the American Chemical Society 117.35 (1995): 9099-9100.

9. Ma J., et al. "Biomimetic processing of nanocrystallite bioactive apatite coating on titanium". Nanotechnology 14.6 (2003): 619.

10. De La Isla Agustin., et al. "Nanohybrid scratch resistant coatings for teeth and bone viscoelasticity manifested in tribology". Materials Research Innovations 7.2 (2003): 110-114.

11. Weissleder Ralph., et al. "Ultrasmall superparamagnetic iron oxide: characterization of a new class of contrast agents for MR imaging". Radiology 175.2 (1990): 489-493.

12. Nam Jwa-Min., et al. "Nanoparticle-based bio-bar codes for the ultrasensitive detection of proteins". Science 301.5641 (2003): 1884-1886.

13. Shanmugaratnam S., et al. "Nanocatalysts" ed. I. Sinha, M. Shukla, Intech Open (2019): 75-96.

14. Niu, H. J., et al. "Critical parameters of disordered nanocrystalline superconducting Chevrel-phase $\mathrm{PbMo}_{6} \mathrm{~S}_{8}$ ". Physical Review B 69.17 (2004): 174503.

15. Dietl Tomasz. "Ferromagnetic semiconductors". Semiconductor Science and Technology 17.4 (2002): 377.

16. Ennaoui A., et al. "Iron disulfide for solar energy conversion". Solar Energy Materials and Solar Cells 29.4 (1993): 289-370.

17. Dutta Amit Kumar., et al. "Synthesis of FeS and FeSe nanoparticles from a single source precursor: a study of their photocatalytic activity, peroxidase-like behavior, and electrochemical sensing of $\mathrm{H}_{2} \mathrm{O}_{2}$ ". ACS Applied Materials and Interfaces 4.4 (2012): 1919-1927.

18. Wood C. "Materials for thermoelectric energy conversion". Reports on Progress in Physics 51.4 (1988): 459.

19. Qi Shihan., et al. "Progress on iron oxides and chalcogenides as anodes for sodium-ion batteries". Chemical Engineering Journal 379 (2020): 122261.

20. kim J., et al. "Identification of marcasite in pyrite FeS2 thin films and the films' carrier transport characteristics". RSC Advances 6 (2016): 81394-81399.

21. Yang Kai., et al. "FeS nanoplates as a multifunctional nanotheranostic for magnetic resonance imaging guided photothermal therapy". Biomaterials 38 (2015): 1-9.
22. Dai Zhihui., et al. "Nanostructured FeS as a mimic peroxidase for biocatalysis and biosensing". Chemistry-A European Journal 15.17 (2009): 4321-4326.

23. Jin Jiayi., et al. "A glassy carbon electrode modified with FeS nanosheets as a highly sensitive amperometric sensor for hydrogen peroxide". Microchimica Acta 184.5 (2017): 13891396.

24. Agnihotri S., et al. "Dual Modality FeS Nanoparticles with Reactive Oxygen Species-Induced and Photothermal Toxicity toward Pathogenic Bacteria". ACS Omega 5 (2020): 597-602.

25. Yadav KK., et al. "Mono Dispersable Water-Soluble Iron Sulfide Nanoclusters: Synthesis, Characterization and Catalytic Application for Highly Efficient Synthesis of Xanthene Derivatives". Advanced Science, Engineering and Medicine 12 (2020): 603611.

26. Jin Liling., et al. "Functionalized graphene oxide in enzyme engineering: a selective modulator for enzyme activity and thermostability". ACS Nano 6.6 (2012): 4864-4875.

27. Zhao Yang., et al. "Efficient Removal of Lead from Water Using Stabilized Iron Sulfide Nanoparticles: Effectiveness and Effects of Stabilizer". Water, Air, and Soil Pollution 230.6 (2019): 1-14.

28. Lyu Honghong., et al. "Immobilization of heavy metals in electroplating sludge by biochar and iron sulfide". Environmental Science and Pollution Research 23.14 (2016): 14472-14488.

29. Watson JHP., et al. "Structural and magnetic studies on heavymetal-adsorbing iron sulphide nanoparticles produced by sulphate-reducing bacteria". Journal of Magnetism and Magnetic Materials 214.1-2 (2000): 13-30.

30. Zhou Lei., et al. "Spectroscopic study on biological mackinawite (FeS) synthesized by ferric reducing bacteria (FRB) and sulfate reducing bacteria (SRB): Implications for in-situ remediation of acid mine drainage". Spectrochimica Acta Part A: Molecular and Biomolecular Spectroscopy 173 (2017): 544548.

31. Xie Yi-Fei., et al. "Study on application of biological iron sulfide composites in treating vanadium-extraction wastewater containing chromium (VI) and chromium reclamation". Journal of Environmental Biology 34.2 (2013): 301.

32. Küsel Kirsten., et al. "Microbial reduction of Fe (III) in acidic sediments: isolation of Acidiphilium cryptum JF-5 capable of coupling the reduction of Fe (III) to the oxidation of glucose". Applied and Environmental Microbiology 65.8 (1999): 36333640. 
33. Huo Ying-Chao., et al. "Biogenic FeS accelerates reductive dechlorination of carbon tetrachloride by Shewanella putrefaciens CN32". Enzyme and Microbial Technology 95 (2016): 236-241.

34. Li Mao-Lin., et al. "Microwave-assisted controlled synthesis of monodisperse pyrite microspherolites". CrystEngComm 13.19 (2011): 5936-5942.

35. Ahuja R., et al. "Synthesis and evaluation of iron (ii) sulfide aqua nanoparticles (FeS-NPs) against Fusarium verticillioides causing sheath rot and seed discoloration of rice". European Journal of Plant Pathology 155.1 (2019): 163-171.

36. Yuan Ye., et al. "Nano-Sized Iron Sulfide: Structure, Synthesis, Properties, and Biomedical Applications". Frontiers in Chemistry 8 (2020): 818.

37. Wu J., et al. "Reactivity enhancement of iron sulfide nanoparticles stabilized by sodium alginate: Taking $\mathrm{Cr}$ (VI) removal as an example". Journal of Hazardous Materials 333 (2017): 275284.

38. Sun Mingyang., et al. "Aqueous Hg (II) immobilization by chitosan stabilized magnetic iron sulfide nanoparticles". Science of the Total Environment 621 (2018): 1074-1083.

39. Yang, Kai, et al. "Multimodal imaging guided photothermal therapy using functionalized graphene nanosheets anchored with magnetic nanoparticles". Advanced Materials 24.14 (2012): 1868-1872.

40. Yang Y., et al. "In vitro and in vivo uncaging and bioluminescence imaging by using photocaged upconversion nanoparticles". Cheng, Angewandte Chemie International Edition 51 (2012): 3125-3129.

41. Yang Kai., et al. "Graphene in mice: ultrahigh in vivo tumor uptake and efficient photothermal therapy". Nano Letters 10.9 (2010): 3318-3323.

42. Dai Zhihui., et al. "Nanostructured FeS as a mimic peroxidase for biocatalysis and biosensing". Chemistry-A European Journal 15.17 (2009): 4321-4326.

43. Yang Kai., et al. "FeS nanoplates as a multifunctional nanotheranostic for magnetic resonance imaging guided photothermal therapy". Biomaterials 38 (2015): 1-9.

44. Xiao Sa., et al. "Iron-mediated activation of persulfate and peroxymonosulfate in both homogeneous and heterogeneous ways: a review". Chemical Engineering Journal 384 (2020): 123265.
45. Gao Lizeng., et al. "Intrinsic peroxidase-like activity of ferromagnetic nanoparticles”. Nature Nanotechnology 2.9 (2007): 577-583.

46. Winter William E., et al. "The molecular biology of human iron metabolism". Laboratory Medicine 45.2 (2014): 92-102.

47. Ludwig Heinz., et al. "Iron metabolism and iron supplementation in cancer patients". Wiener Klinische Wochenschrift 127.23 (2015): 907-919.

48. Torti., et al. "Iron and cancer: more ore to be mined". Nature Reviews Cancer 13.5 (2013): 342-355.

49. Chen., et al. "Tumor-induced disorder of iron metabolism in major organs: a new insight from chemical speciation of iron". Journal of International Medical Research 46.1 (2018): 70-78.

50. Chang., et al. "Fabrication, characterization, and application of greigite nanoparticles for cancer hyperthermia". Journal of Colloid and Interface Science 363.1 (2011): 314-319.

51. Yang Kai., et al. "FeS nanoplates as a multifunctional nanotheranostic for magnetic resonance imaging guided photothermal therapy". Biomaterials 38 (2015): 1-9.

52. Ning S., et al. "Laser-triggered combination therapy by iron sulfide-doxorubicin@functionalized nanozymes for breast cancer therapy. Journal of Nanobiotechnology 19.344 (2021).

53. Guan Guoqiang., et al. "“Transformed" Fe 3 S 4 tetragonal nanosheets: a high-efficiency and body-clearable agent for magnetic resonance imaging guided photothermal and chemodynamic synergistic therapy". Nanoscale 10.37 (2018): 17902-17911.

54. Dai Zhihui., et al. "Nanostructured FeS as a mimic peroxidase for biocatalysis and biosensing". Chemistry-A European Journal 15.17 (2009): 4321-4326.

\section{Assets from publication with us}

- Prompt Acknowledgement after receiving the article

- Thorough Double blinded peer review

- Rapid Publication

- Issue of Publication Certificate

- High visibility of your Published work

Website: www.actascientific.com/

Submit Article: www.actascientific.com/submission.php

Email us: editor@actascientific.com

Contact us: +919182824667 